\title{
Electron Diffraction Pattern of Crystal Foils Containing a High Density of Point-Defect Agglomerates
}

\author{
By M. Wilkens, K. Urban and K.-H. Katerbau \\ Max-Planck-Institut für Metallforschung, Institut für Physik, Stuttgart, Germany (BRD)
}

(Received 4 June 1974)

Using high-voltage electron microscopy at low temperatures $(\mathrm{T} \simeq 10-30 \mathrm{~K})$ Urban, K. [J. Microsc. (1973). 97, 121] has shown that during irradiation at voltages above the threshold voltage for atomic displacements an extremely high density of small point-defect agglomerates (PDA) is produced in nickel and copper specimens. If the nominal displacements per atom (d.p.a.) exceed about $5 \times 10^{-2}$ the contrasts of the PDA overlap to such an extent that they can no longer be resolved on the micrographs. At the same time the diffraction pattern of the specimen is modified: The diffracted intensity, which at the beginning is strongly concentrated in the low-order reflexions (including the zero-order beam), becomes nearly equipartitioned over a number $N$ of reflexions, where $N$ increases with increasing d.p.a. It is suggested that this effect may be useful for an estimate of the number of point defects stored in the PDA at high doses where any direct counting and measuring of the PDA is impossible. The paper deals with a brief outline of a theoretical approach to this problem, in which the dynamic interactions of the $N$ beams involved are taken into account.

J. Appl. Cryst. (1975). 8, 229

\section{Interatomic Forces and the Theory of Local Distortions*}

\author{
By W. A. HARrison \\ Stanford University, Stanford, California 94305, U.S.A.
}

(Received 29 April 1974)

\begin{abstract}
The relation between lattice distortions in metals and the corresponding interatomic interactions is discussed, with special attention to the fundamental questions which can be answered through experimental studies of the distortions. By the expansion of the total energy of a metal in terms of pseudopotentials, the contributions can be classified as one-body, two-body, three-body, etc. interactions [Harrison, W. A. (1973). Phys. Rev. B 7, 2408]. The most familiar form of the two-body interaction is discussed and seen, because of possible phase-shift effects, to be inadequate. The consequences of this failure are illustrated in terms of distortions near point defects. The form of multi-body interactions is displayed and seen to be largest when many atoms form straight lines. This feature can show up in the local atomic arrangements in ways which would not be expected on the basis of the more intuitive twobody model. Such interactions are certainly real and their general magnitude significant and known, but the role they play can only be settled with experimental information.
\end{abstract}

* Supported by the U. S. Army Research Office. 